\title{
Takotsubo Syndrome Associated with COVID-19
}

\author{
Elena Roca ${ }^{1,2}$, Carlo Lombardi ${ }^{1,3}$, Marco Campana ${ }^{4}$, Oscar Vivaldi ${ }^{1}$, Barbara Bigni $^{1}$, Bruno Bertozzi ${ }^{1}$, Giovanni Passalacqua ${ }^{5}$ \\ ${ }^{1}$ COVID-19 Unit, Istituto Ospedaliero Poliambulanza, Brescia, Italy \\ ${ }^{2}$ Technology for Health, PhD program, University of Brescia, Italy \\ ${ }^{3}$ Pulmonary and Allergic Respiratory Diseases Department, Poliambulanza Foundation Hospital, Brescia, Italy \\ ${ }^{4}$ Cardiovascular Department, Poliambulanza Foundation Hospital, Brescia, Italy \\ ${ }^{5}$ Allergy and Respiratory Diseases, IRCCS Policlinico San Martino - University of Genoa, Italy
}

Received: $16 / 04 / 2020$

Accepted: $17 / 04 / 2020$

Published: $21 / 04 / 2020$

How to cite this article: Roca E, Lombardi C, Campana M, Vivaldi O, Bigni B, Bertozzi B, Passalacqua G. Takotsubo syndrome associated with COVID-19. EJCRIM 2020;7: doi:10.12890/2020_001665.

Conflicts of Interests: The Authors declare that there are no competing interests.

This article is licensed under a Commons Attribution Non-Commercial 4.0 License

\section{ABSTRACT}

Objective: The availability of public health information for optimised supportive care is critical during the COVID-19 pandemic. We describe the first case of COVID-19 complicated by Takotsubo cardiomyopathy.

Materials and Methods: We report the clinical, laboratory and radiological findings of a patient with severe acute respiratory syndrome coronavirus 2 (SARS-CoV-2).

Results: The nasopharyngeal swab was positive for SARS-CoV-2 and x-ray images demonstrated pathognomonic pneumonia. The patient developed tachycardia and the echocardiogram confirmed the diagnosis of Takotsubo cardiomyopathy.

Conclusions: Doctors should be aware of the need to thoroughly study this new infection in order to understand its underlying mechanisms and related complications.

\section{LEARNING POINTS}

- We report the first case of Takotsubo cardiomyopathy associated with COVID-19.

- We discuss a rare presentation in the current pandemic.

- COVID-19 can be associated with cardiac complications, even after the onset of pneumonia, and so strict monitoring of these patients is essential.

\section{KEYWORDS}

SARS-CoV-2, severe acute respiratory syndrome coronavirus 2, coronavirus, COVID-19, Takotsubo syndrome

\section{BACKGROUND}

On 31 December 2019, the Wuhan Municipal Health Commission reported to the World Health Organization (WHO) a cluster of cases of pneumonia of unknown aetiology in the city of Wuhan, in the Chinese province of Hubei. On 9 January 2020, the Chinese Center for Disease Control and Prevention reported that a new coronavirus had been identified as the causative agent of the respiratory disease. The International Committee on Taxonomy of Viruses (ICTV) named the new coronavirus 'severe acute respiratory syndrome coronavirus 2' (SARS-CoV-2) and on 11 February the WHO officially called it COVID-19. Since the beginning of the epidemic, 185 countries have been affected and currently more than 2.2 million cases have been confirmed globally, with 156,064 deaths (Johns Hopkins University data). The Ministry of Health in Italy announced tight restrictions for most of the population on 11 March. 
We describe a rare cardiac complication, Takotsubo syndrome, in a patient infected with SARS-CoV-2, in the hope of increasing understanding of the severity and possible complications of COVID-19 and improving clinical management.

The patient provided written informed consent and the diagnostic procedures were conducted in accordance with institutional guidelines on the protection of human subjects.

\section{CASE DESCRIPTION}

An 87-year-old woman was admitted to our clinic on 24 March 2020, with fever, fatigue and shortness of breath. She had a history of breast cancer, but no hypertension, diabetes or obstructive pulmonary disease. She reported no travel to China or possible contact with infected individuals. She had experienced chills and a dry cough 2 weeks previously but had not sought medical advice. A chest $\mathrm{x}$-ray showed multiple patchy shadows in both lungs and parenchymal thickening with bilateral basal alveolar interstitial infiltrates compatible with COVID-19 (Fig. 1). A nasopharynx swab sample was taken. Using reverse real-time PCR assay, the laboratory confirmed on 25 March that the patient was positive for SARS-CoV-2.

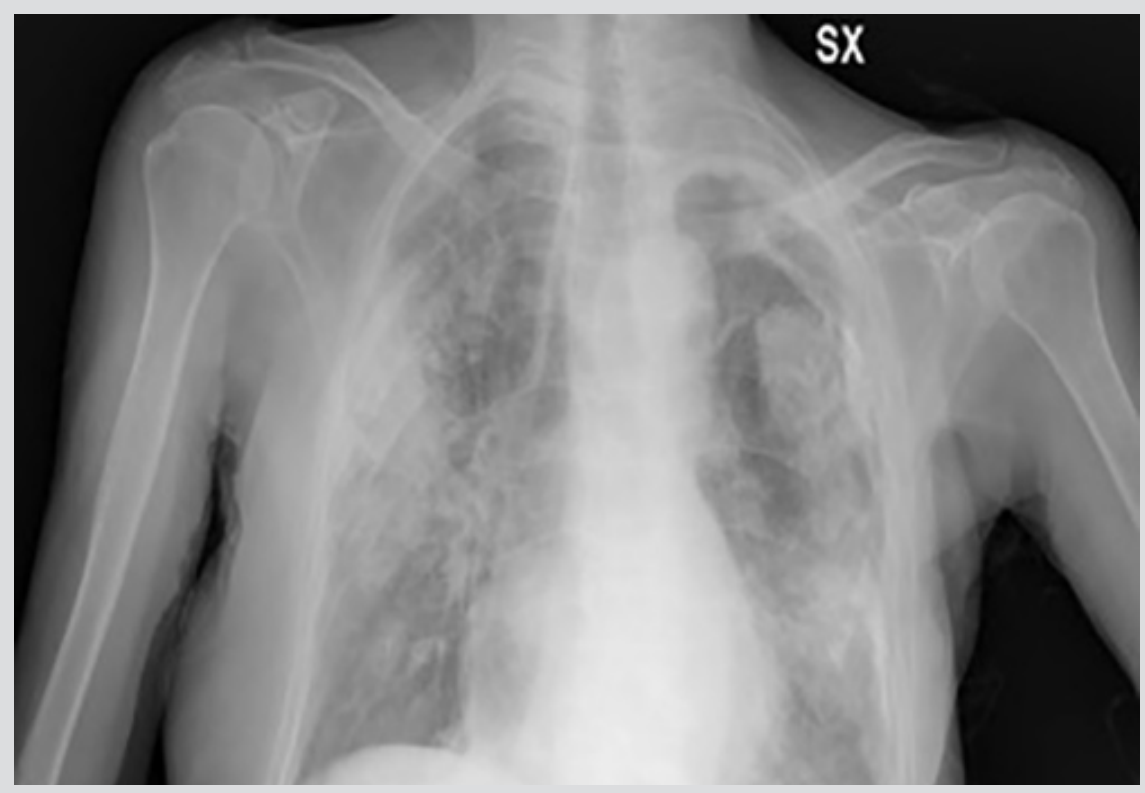

Figure 1. Chest $x$-ray showing multiple patchy shadows in both lungs and parenchymal thickening with bilateral basal alveolar interstitial infiltrates

She was immediately admitted to the isolation ward and received supplemental oxygen through a face mask (12 I/min with a $50 \%$ Ventimask), achieving an oxygen saturation of $91 \%$. She was also treated with azithromycin (500 mg once daily, orally) and ceftriaxone (2 g once daily, intravenously). Given the serious shortness of breath and hypoxaemia, methylprednisolone (20 mg once daily, intravenously) was administered to reduce lung inflammation.

Laboratory test results showed 1.17\% lymphocytes (normal range 20-50\%), 95.8\% neutrophils (normal range 41-70\%) and C-reactive protein of 205.6 (normal value <5). Oxygen pressure was $47 \mathrm{mmHg}$ (normal range 83-108) with a $\mathrm{PO}_{2} / \mathrm{FO}_{2}$ of 226 (normal value $>300$ ) On the second day of hospitalisation, the patient had an episode of tachycardia with an increase in cardiac laboratory markers: troponin I was $5318 \mathrm{ng} / \mathrm{I}$ (normal value <6) and CK-MB was 55 gg/I (normal value <3). The electrocardiogram showed negative T waves and repolarization phase alterations. An echocardiogram was therefore performed and showed alterations in the left ventricle: apical akinetic expansion (apical ballooning) and hypokinesia of the mid-ventricular segments with slightly reduced systolic function (ejection fraction slightly reduced to 48\%) (Fig. 2). The clinical, laboratory and radiological picture was diagnostic for Takotsubo syndrome. Due to the age of the patient, coronary angiography was not possible but treatment with bisoprolol and fondaparinux was started.

The patient was discharged in good clinical conditions.

\section{DISCUSSION}

Takotsubo syndrome was first described in 1991 by Dote et al ${ }^{[1]}$ in Japan. The Japanese term was taken from the name of a fishing pot with a round bottom and a narrow neck used for trapping octopus. There have been some suggestions regarding the pathogenesis of this syndrome, but the causes remain unknown ${ }^{[2]}$. 


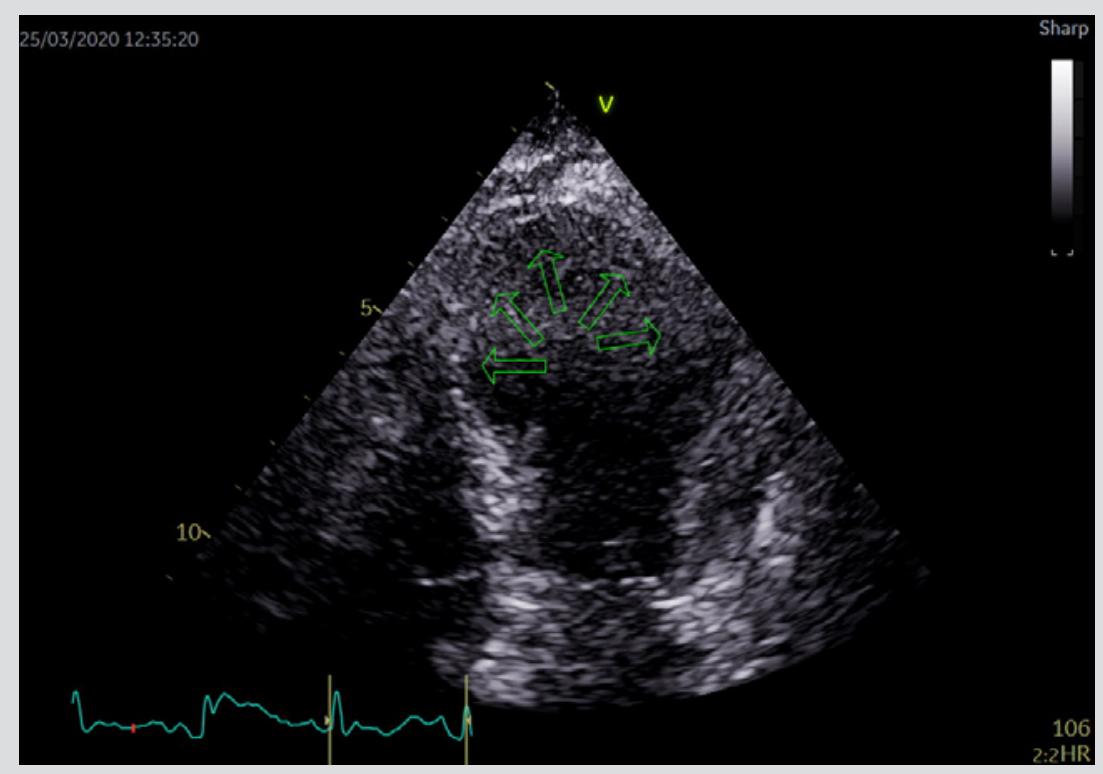

Figure 2. Echocardiogram (telesystolic phase) showing alterations in the left ventricle: apical akinetic expansion (apical ballooning) and hypokinesia of the mid-ventricular segments with slightly reduced systolic function

Some studies have demonstrated the presence of viral infections by endomyocardial biopsy in patients with left ventricular dysfunction of unknown cause. In particular, Kühl et al. reported a high frequency of a wide spectrum of cardiotrophic viruses, with genomes detectable in $71 \%$ of their patients ${ }^{[3]}$. Parvovirus B19 was the most frequent pathogen, at $50 \%$, as also described by Pankuweit et al. ${ }^{[4,5]}$.

Viral infections can cause degeneration and necrosis of myocytes, myocarditis and congestive heart failure, and are also implicated in the development of idiopathic dilated cardiomyopathy ${ }^{[6]}$.

We describe what may be the first case of Takotsubo syndrome caused by a coronavirus infection. About a quarter of patients with pneumonia develop cardiac complications during hospitalisation, about $90 \%$ in the first weeks after diagnosis and more than half within 24 hours. Currently, $19.7 \%$ of COVID-19 patients have associated cardiac complications, although Takotsubo syndrome has not been previously described ${ }^{[7]}$.

This virus may damage vascular integrity and cause the myocardial abnormalities observed in Takotsubo syndrome. Some authors have reported cases of Takotsubo syndrome associated with CMV infection: the virus may mediate coronary endothelial dysfunction with consequent increased expression of endothelial adhesion molecules and transendothelial migration within the vasculature. SARS-CoV-2 may exert a direct toxic action on myocytes; indeed the SARS-CoV-2 spike protein shows high-affinity binding to angiotensin-converting enzyme 2 (ACE2), a human cell receptor which is highly expressed in the heart ${ }^{[8]}$.

In the present case, the patient may have experienced stress-induced adrenergic discharge due to fever and to the inflammatory response to infection. This, in addition to the direct SARS-CoV-2 damage with endothelial dysfunction, may have caused microvascular vasoconstriction that could have resulted in transient left ventricular apical dysfunction, so-called 'apical ballooning' (apical akinetic expansion with hypokinesia of the mid-ventricular segments).

\section{CONCLUSIONS}

We report the first case described in the literature of a COVID-19 patient with associated Takotsubo syndrome.

Several mechanisms have been hypothesized as possible causes of this syndrome. In our case, greater myocardial reactivity to sympathetic stimulation (caused indirectly by pneumonia) in the apical region combined with abnormal vascular reactivity (induced directly by the virus) could have determined the onset of the left ventricular dysfunction seen in Takotsubo syndrome.

Acute management of this cardiac condition, especially in elderly patients, may be difficult if the diagnosis is late. Therefore, SARSCoV-2 should be considered in patients presenting with Takotsubo syndrome to further investigate the role of viral infections in the pathophysiological mechanisms responsible for this syndrome. As patients with cardiac complications during COVID-19 have a higher mortality rate, we believe that early diagnosis of this disease is important in order to improve patient outcome. 


\section{REFERENCES}

1. Dote KSH, Tateishi H, Uchida T, Ishihara M. Myocardial stunning due to simultaneous multivessel coronary spasm: a review of 5 cases. J Cardiol 1991;21:203-14.

2. Stollberger CFJ, Schneider B. Tako-tsubo-like left ventricular dysfunction: clinical presentation, instrumental findings, additional cardiac and non-cardiac diseases and potential pathomechanisms. Minerva Cardioangiol 2005;53:139-145.

3. Kühl U, Noutsias M, Seeberg B et al. High prevalence of viral genomes and multiple viral infections in the myocardium of adults with "idiopathic" left ventricular dysfunction. Circulation 2005;111.

4. Pankuweit S, Eckhardt H, Strache D, Maisch B. Pathophysiology and aetiological diagnosis of inflammatory myocardial diseases with a special focus on parvovirus B19. J Vet Med 2005;B52:344-7.

5. Bahlmann E, Schneider C, Krause K, et al. Tako-Tsubo cardiomyopathy (apical ballooning) with parvovirus B19 genome in endomyocardial biopsy. Int J Cardiol 2007;116(1):e1821.

6. Edwards S, Small JD, Geratz JD, et al. An experimental model for myocarditis and congestive heart failure after rabbit coronavirus infection. J Infect Dis 1992;165(1):134-140.

7. Shi S, Qin M, Shen B, et al. Association of cardiac injury with mortality in hospitalized patients with COVID-19 in Wuhan, China. JAMA Cardiol 2020 Mar 25. doi: 10.1001/ jamacardio.2020.0950 [Epub ahead of print].

8. Greco C, Saolini M, Mariani SJ, et al. Tako-tsubo syndrome: a potential role for cytomegalovirus infection. Cardiovasc Med (Hagerstown) 2006 ;7(8):623-627. 